\title{
SOCIETY PRIZES
}

\section{Canadian Neurological Society announces the}

\section{FRANCIS McNAUGHTON MEMORIAL PRIZE and the}

\section{ANDRÉ BARBEAU MEMORIAL PRIZE}

The Canadian Neurological Society invites submissions for its two prizes: the Francis McNaughton Memorial Prize for clinical research and the André Barbeau Memorial Prize for basic research.

The Francis McNaughton and André Barbeau Memorial Prizes, designed to encourage Neurology Trainees to undertake research projects, are awarded for the best submitted papers based on work done during the Neurology residency or in post-residency training. The contestants need not be the sole authors but should have been primarily responsible for the work to be presented.

The winning papers in each category will be presented at the annual meeting of the Canadian Congress of Neurological Sciences. The deadline for submission is December 31, 1994.

\section{Canadian Society of Clinical Neurophysiologists}

announces

\section{THE HERBERT JASPER PRIZE}

The Herbert Jasper Prize is awarded annually for the best submitted paper in clinical or basic neurophysiology by a resident or fellow in training. Others who are within three years of receiving a doctorate or fellowship are also eligible.

The prize consists of an honorarium of $\$ 250.00$, economy air transportation, hotel accommodation, and registration to attend the annual meeting of the Canadian Congress of Neurological Sciences where the winning paper will be presented.

The deadline for submission is December 31, 1994.

\author{
The Royal College of \\ Physicians and Surgeons of Canada \\ with the co-operation of the \\ Canadian Neurosurgical Society
}

\author{
announces the
}

\section{K.G. McKENZIE MEMORIAL PRIZE AND AWARD}

A. The McKenzie Prize for Basic Neurosciences Research and the McKenzie Prize for Clinical Neuroscience Research There will be one citation and prize of $\$ 1,000.00$ in each of the Basic Neuroscience and Clinical Neuroscience categories for the best manuscripts submitted to the McKenzie Award Committee of the Canadian Neurosurgical Society, by a neurosurgical resident, in which he or she is the principal author. The recipients will have their expenses, including air fare, hotel accommodation and registration fees, paid for as part of the Prizes. A second prize of $\$ 500.00$ may also be awarded in each category.

B. The McKenzie Training or Research Award

A grant will be awarded to support specific training or research which may include travel. However, travel to regularly scheduled neurosurgical meetings is excluded. The applicant must be resident in training in a Canadian Neurosurgical Training Program, or if formal training is completed, within two years of having received certification for Fellowship in neurosurgery. The amount to be awarded will be up to $\$ 2,000.00$.

The deadline for the Prize and Award for 1995 is December 15, 1994.

\author{
The Canadian Association for \\ Child Neurology \\ announces
}

\section{THE PRESIDENT'S PRIZE}

The President's Prize is awarded annually for the best paper in pediatric neuroscience by a resident or fellow in training. The prize consists of a $\$ 500.00$ honorarium and a commemorative scroll. The winning paper will be presented at the annual meeting of the Canadian Congress of Neurological Sciences.

Deadline for receipt of submissions is December 31, 1994. 


\section{PRIX DES SOCIÉTÉS}

\author{
Prix de la Société Canadienne de Neurologie
}

\section{PRIX COMMÉMORATIF FRANCIS MCNAUGHTON et la prix COMMÉMORATIF ANDRÉ BARBEAU}

La Société canadienne de neurologie accepte des soumissions de communications pour ses deux prix : le prix commémoratif Francis McNaughton pour la recherche clinique et le prix commémoratif André Barbeau pour la recherche fondamentale.

Les prix commémoratifs Francis McNaughton et André Barbeau ont pour but d'encourager les résidents en neurologie à entreprendre des projets de recherche. Ils sont décernés aux meilleures communications pour un travail exécuté au cours d'un programme de formation en résidence ou post-résidence en neurologie. Il n'est pas nécessaire que les candidats soient les seuls auteurs, mais ils doivent être les principaux responsables de la communication présentée.

Les communications gagnantes dans chaque catégorie seront présentées àl'assemblée annuelle du Congrès canadien des sciences neurologiques. La date limite pour la soumission des communications est fixée au 31 décembre 1994.

\section{Société Canadienne de neurophysiologie clinique}

\section{LE PRIX HERBERT JASPER}

Le prix Herbert Jasper est décerné chaque année au résident ou au chercheur qui a soumis la meilleure communication dans le domaine de la neurologie fondamentale ou clinique. Ceux qui doivent obtenir leur doctorat ou leur bourse de recherche d'ici trois ans sont aussi admissibles.

Le prix comprend une somme de 250 \$, l'hébergement à l'hôtel, les frais d'inscription, et un billet d'avion en classe économique pour assister au Congrès canadien des sciences neurologiques où sera présentée la communication gagnante.

La date limite des soumissions est fixée au 31 décembre 1994.

\author{
Le Collège royal des médecins et \\ chirurgiens du Canada \\ en collaboration avec la \\ Société canadienne de neurochirurgie \\ présente
}

\section{LES PRIX COMMÉMORATIFS}

K. G. McKENZIE 1995

A. Le prix McKenzie pour la recherche fondamentale en sciences neurologiques et le prix McKenzie pour la recherche clinique en sciences neurologiques

Dans chacune des catégories de la recherche fondamentale et de la recherche clinique en sciences neurologiques, on attribuera une mention et un prix de $1000 \$$ au(à la) résident(e) en neurochirurgie qui est le principal auteur du meilleur manuscrit soumis au comité du prix McKenzie de la Société canadienne de neurologie. Les prix comprendront également les dépenses des lauréats, y compris le transport aérien, l'hébergement à l'hôtel et les frais d'inscription. Un deuxième prix de $500 \$$ pourrait également être remis dans chaque catégorie.

B. Le prix McKenzie pour la formation ou la recherche

Une subvention sera accordée en vue d'appuyer un programme de formation ou une recherche précise, qui pourrait comporter des déplacements. Cependant, les frais de déplacements pour des réunions de neurochirurgie qui ont lieu à intervalles réguliers sont exclus. Le candidat doit être un résident inscrit à un programme de formation en neurochirurgie au Canada ou, si sa formation conventionnelle est terminée, il doit se trouver à moins de deux ans de l'obtention de sa bourse de recherche en neurochirurgie. La somme attribuée pourrait atteindre $2000 \$$.

La date limite des soumissions pour les prix de 1995 est fixée au 15 décembre 1994.

\section{L'Association Canadienne de neurologie pédiatrique \\ LE PRIX DU PRÉSIDENT}

Le prix du président est décerné chaque année au résident ou au chercheur qui a soumis la meilleure communication dans le domaine de la neuroscience chez l'enfant. Le prix comprend une somme de $500 \$$ et un parchemin commémoratif. La communication gagnante sera présentée à l'assemblée du Congrès canadien des sciences neurologiques.

La date limite de la réception des soumissions est fixée au 31 décembre 1994. 